\title{
Texture classification using discrete Tchebichef moments
}

\author{
J. Víctor Marcos* and Gabriel Cristóbal \\ Instituto de Óptica (CSIC), Serrano 121, 28006 Madrid, Spain \\ *Corresponding author: jvmarcos@gmail.com
}

Received January 30, 2013; revised June 5, 2013; accepted June 7, 2013; posted July 2, 2013 (Doc. ID 184450); published July 17, 2013

In this paper, a method to characterize texture images based on discrete Tchebichef moments is presented. A global signature vector is derived from the moment matrix by taking into account both the magnitudes of the moments and their order. The performance of our method in several texture classification problems was compared with that achieved through other standard approaches. These include Haralick's gray-level co-occurrence matrices, Gabor filters, and local binary patterns. An extensive texture classification study was carried out by selecting images with different contents from the Brodatz, Outex, and VisTex databases. The results show that the proposed method is able to capture the essential information about texture, showing comparable or even higher performance than conventional procedures. Thus, it can be considered as an effective and competitive technique for texture characterization. (C) 2013 Optical Society of America

OCIS codes: (100.2000) Digital image processing; (100.5010) Pattern recognition; (150.1135) Algorithms. http://dx.doi.org/10.1364/JOSAA.30.001580

\section{INTRODUCTION}

Texture has been a subject of study for the image processing community during the last decades. However, there is no consensus about its definition. Tuceryan and Jain [1] compiled the definitions provided by different researchers. According to them, texture is a property related to the roughness of a surface and is characterized by the variation of pixel intensity in the spatial domain. Texture can be found in images of different nature: for instance, a specific body tissue, an area of terrain, the surface of an object, or the skin of a person or an animal. Thus, an accurate characterization of texture properties is required in different real-world applications involving image processing. Specifically, texture plays a relevant role in tasks such as medical diagnosis [2] , remote sensing [3] , or biometric identification [4].

Mainly, texture analysis deals with four types of tasks: texture classification, texture edge identification, texture synthesis, and the shape-from-texture problem [1]. The former is addressed in the present study. Texture classification aims to automatically identify image regions characterized by different texture properties. Thus, the attributes that differentiate each texture from the other ones must be captured. Several methods have been proposed in the literature to perform texture classification. They are conventionally grouped into four categories [1] : (1) statistical methods, (2) geometrical (structural) approaches, (3) model-based techniques, and (4) signal processing methods based on spatial- and frequency-domain filtering. The latter represent a traditional approach to texture characterization [5]. Experimental results suggest that the human brain identifies textures by means of space-frequency analysis of the input image [6]. As a result, this behavior has been simulated through signal processing techniques based on image filtering. Indeed, a number of studies focused on texture classification propose frequency-based and spatial frequency-based analysis of the image. For instance, filter banks [7], wavelets [8], and Gabor filters [9,10] have proven to be valid techniques for automatic texture identification, showing high classification performance.

Closely related to signal processing techniques, image moments have been widely used for pattern recognition tasks, and they suitably adapt to texture analysis [11]. It is worth noting the pioneering work on the construction of moment invariants performed by $\mathrm{Hu}$ [12] in the 1960s. Moments are scalar quantities used to characterize a function (image), reflecting significant attributes of it. Mathematically, the moment $T_{p q}$ of an image $f(x, y)$, where $p$ and $q$ are nonnegative integers and $s=p+q$ denotes its order, is computed from the projection of the image onto a polynomial basis $r_{p q}(x, y)$ defined on a region $D: T_{p q}=\iint_{D} r_{p q}(x, y) f(x, y) \mathrm{d} x \mathrm{~d} y$ [11]. Thus, the value of $T_{p q}$ measures the correlation between the image $f(x, y)$ and the corresponding polynomial function $r_{p q}(x, y)$. Several moment families have been defined in the literature by using different polynomial bases. They can be grouped as nonorthogonal and orthogonal depending on the behavior of the basis functions. The former include geometric and complex moments, which represent a conventional approach for image analysis [11]. Indeed, these moments were previously used for feature extraction from texture images $[13,14]$. However, as the basis set used to compute geometric and complex moments is nonorthogonal, a redundant representation of the information contained in the image is obtained [15]. Additionally, nonorthogonality causes the problem of image recovery from these moments to be strongly ill-posed and computationally expensive [16]. Continuous orthogonal moments such as Legendre and Zernike families were introduced to overcome these drawbacks $[\underline{2}, \underline{17}, \underline{18}]$. Nevertheless, practical implementations of continuous moments lead to two main sources of error. First, their exact definition involves the 
calculation of integrals that are approximated by summations in the discrete domain. Second, Legendre and Zernike polynomials are defined in a specific region of the space. It is given by the interval between -1 and 1 for Legendre polynomials, while Zernike functions are defined in the unit circle. Thus, the image coordinate space must be transformed for their computation $[\underline{15}, \underline{19}]$.

Discrete orthogonal moments avoid these limitations and hold most of the useful properties of continuous orthogonal moments. This is achieved by defining a set of orthogonal polynomials in the image space. As a result, the discretization error inherent to continuous moments is prevented while enabling accurate image reconstruction [19]. Commonly, discrete Tchebichef [15] and Krawtchouk [20] polynomials are used to compute discrete orthogonal moments. For a digital image $f(x, y)$ with $0 \leq x, y \leq N-1$, a total of $N^{2}$ moments can be extracted. The entire moment set is required for a complete description of the image, that is, to ensure perfect image reconstruction. Nevertheless, computation of high-order moments is affected by numerical instabilities motivated by the recurrence equations used to obtain polynomial coefficients [11]. As a result, numerical errors are propagated and accumulated with increasing moment order and image size [19]. To avoid this problem, Mukundan [21] defined scaled Tchebichef polynomials. They are obtained by normalizing Tchebichef polynomials by a factor that depends on their order. The computational approach suggested by Mukundan allows the complete set of discrete Tchebichef moments (DTM) characterizing an image to be computed.

In this study, a novel method based on DTM is proposed to characterize digitized texture images. DTM can yield relevant texture features since they represent the outputs from the filters implemented by Tchebichef kernels [22]. The oscillating shape of these functions reflects the correspondence between frequency and moment order, with higher-frequency components being linked to higher-order moments [22,23]. In our method, only the magnitude of the moments was considered to perform texture feature extraction. It provides information about the similarity of the input image and the polynomial kernel. Hence, higher magnitude is expected for those moments derived from kernel functions showing fluctuating patterns similar to the texture. We propose a signature vector $M(s)$ to capture texture attributes by assessing the total magnitude of the moments of order $s$. As shown in our study, $s$-order Tchebichef kernels are characterized by the same oscillating pattern along different directions. Thus, the value of $M(s)$ reflects the occurrence of this pattern in the underlying texture. The dependence of the proposed feature on the moment order enables the interpretation of texture properties. For instance, for a high-order $s$, an increased value of $M(s)$ indicates that texture contains rapidly varying patterns. The plot of $M(s)$ versus $s$ can be used for a qualitative evaluation of the texture. In addition, $M(s)$ enables a notable reduction of dimensionality [24]. Given a $N \times N$ image, the initial moment set composed of $\overline{N^{2}}$ coefficients is mapped onto a vector of dimension $2 N-1$.

Previously, DTM have been applied to problems such as image quality assessment [22,25], digital watermarking [26], image autofocus [23], or image compression [27]. To our knowledge, only a reduced number of studies have been focused on the use of DTM to describe texture features. These were basically devoted to texture analysis in medical applications. In particular, low-order DTM were suggested to characterize color texture features in gastrointestinal-tract images from endoscopy [28]. In a different vein, a combination of several moment families, including DTM, was proposed to represent texture regions in CT liver images [29]. Moments up to order four were considered for regions of size $21 \times 21$ pixels. On the other hand, discrete cosine transform (DCT) coefficients, which are computed from a polynomial basis similar to that of DTM [30], have been used for texture characterization [31]. DCT-based analysis was conceived as an effective approach for processing JPEG compressed images. For this purpose, image processing is carried out on $8 \times 8$ blocks as defined by the standard [31].

The aim of this study is to assess the utility of the proposed DTM-based method for automatic identification of texture images. A supervised classification approach is used to model this task. Thus, given a texture image, the goal is to determine its category from a finite number of possible choices. Regularized discriminant analysis (RDA) [32] is proposed to implement the classification stage. Texture images from several public databases including Brodatz [33], Outex [34], and VisTex [35] have been used to validate our methodology. An exhaustive evaluation of the method is presented, analyzing the influence of the image size and rotation on classification performance. For a fair validation, several well-known techniques for texture analysis, such as Haralick's gray-level cooccurrence matrices (GLCM) [36], Gabor filters (GF) [37,38], and local binary patterns (LBP) [39] were used as a reference. A comparative analysis of the results achieved by these methods and the approach presented in this study is provided.

\section{METHODS}

Texture classification involves two main tasks: feature extraction and classification [36]. The present study is focused on the former. A novel feature is proposed to represent texture properties. In order to assess its utility, it was compared with other methods that have been proven to be effective in texture feature extraction: GLCM, GF, and LBP. For a fair comparison, the same classification approach was used to process the feature vectors derived from each of these methods. RDA developed by Friedman [32] was selected for this purpose since it suitably adapts to both high- and lowdimensional input feature spaces. A detailed description of the DTM-based method for texture analysis is presented in this section.

\section{A. Texture Signature Based on DTM}

DTM were introduced by Mukundan et al. [15] in order to overcome the limitations of conventional orthogonal moments based on Legendre and Zernike polynomials. DTM are computed by projecting the original image $f(x, y) \quad(x$, $y=0,1, \ldots, N-1)$ onto a set of Tchebichef polynomial kernels, which include basis functions of the DCT as a special case [30]. The moment $T_{p q}(p, q=0,1, \ldots, N-1)$ of order $s=$ $p+q$ is defined as [15]

$$
T_{p q}=\frac{1}{\tilde{\rho}(p, N) \tilde{\rho}(q, N)} \sum_{x=0}^{N-1} \sum_{y=0}^{N-1} \tilde{t}_{p}(x) \tilde{t}_{q}(y) f(x, y),
$$

where $\tilde{t}_{n}(x, N)$ is the scaled Tchebichef polynomial of degree $n$ and $\tilde{\rho}(n, N)$ is its squared norm. The polynomial $\tilde{t}_{n}(x, N)$ is a 
modified version of the discrete Tchebichef polynomial $t_{n}(x, N)$ by including a scale factor $\beta(n, N)$ :

$$
\tilde{t}_{n}(x, N)=t_{n}(x, N) / \beta(n, N) .
$$

Mukundan [21] proposed a computational procedure to ensure numerical stability of DTM even in the case of large values of the polynomial degree $n$ and/or the image dimension $N \times N$. It requires using the following value for the scale factor:

$$
\beta(n, N)=\left[\frac{N\left(N^{2}-1\right)\left(N^{2}-2^{2}\right) \cdots\left(N^{2}-n^{2}\right)}{2 n+1}\right]^{1 / 2},
$$

which results in an orthonormal basis of scaled Tchebichef polynomials $\tilde{t}_{n}(x, N)$, that is, $\tilde{\rho}(n, N)=1$. The orthogonality property of the obtained basis ensures exact image reconstruction from the set of moments $T_{p q}$ :

$$
f(x, y)=\sum_{p=0}^{N-1} \sum_{q=0}^{N-1} T_{p q} \tilde{t}_{p}(x) \tilde{t}_{q}(y) .
$$

According to Eq. (1), the value of $T_{p q}$ can be interpreted as the correlation between the image $f(x, y)$ and the kernel function $r_{p q}(x, y)$ built from discrete Tchebichef polynomials, which is given by

$$
r_{p q}(x, y)=\frac{1}{\tilde{\rho}(p, N) \tilde{\rho}(q, N)} \tilde{t}_{p}(x) \tilde{t}_{q}(y)
$$

These kernel functions are characterized by an oscillating behavior, showing a sine-like profile. Figure 1 shows the set of Tchebichef kernels for $N=8$ in both spatial and frequency domains. As can be observed, as the order of the kernel increases, the energy of the kernel function tends to be concentrated in higher frequencies [22].

The kernel $r_{p q}(x, y)$ acts as a filter for the computation of $T_{p q}$. The magnitude of $T_{p q}$ will be higher for images oscillating at a similar rate to $r_{p q}(x, y)$ along both directions. This is an interesting characteristic for texture analysis since texture involves the spatial repetition of intensity patterns [1]. Thus, a description of texture properties can be obtained by assessing the dependence of the moment magnitude on the order $s$, which is related to the frequency content of the kernels. For this purpose, the following feature vector $M(s)$ $(s=0,1, \ldots, 2 N-2)$ is proposed:

$$
M(s)=\sum_{p+q=s}\left|T_{p q}\right| .
$$

The feature $M(s)$ provides information about the properties of the texture and can be viewed as a texture signature. To evaluate the specific attributes captured by $M(s)$, the behavior of Tchebichef kernels in both spatial and frequency domains is studied.

\section{Spatial Analysis of Tchebichef Moments}

The magnitude of the moment $T_{p q}$ with order $s=p+q$ quantifies the correlation between the signal $f(x, y)$ and the Tchebichef kernel $r_{p q}(x, y)$. As described before, $T_{p q}$ is obtained by projecting $f(x, y)$ onto $r_{p q}(x, y)$, which is characterized by a sinusoidal profile. This process involves

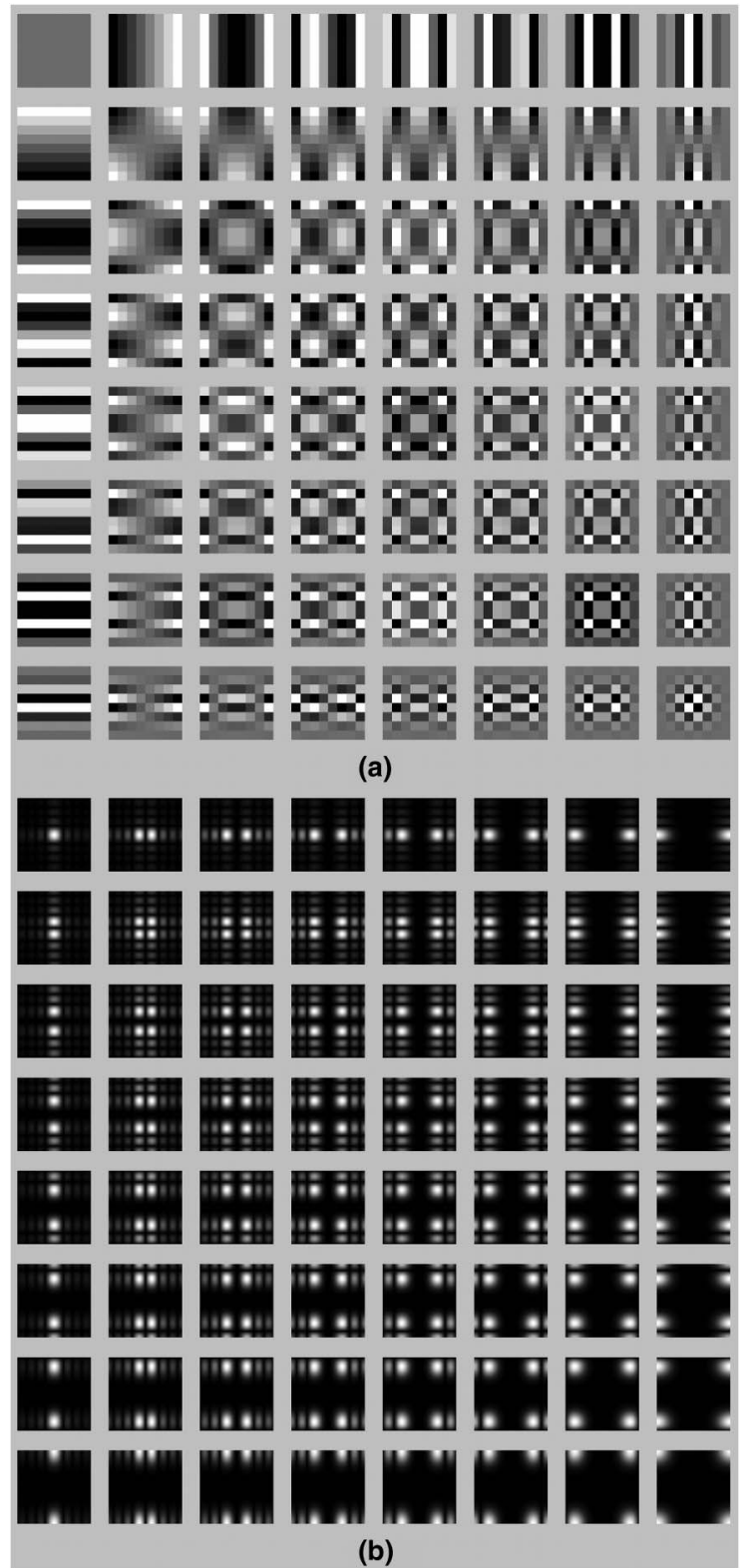

Fig. 1. Complete set of Tchebichef kernels $(N=8)$ in both (a) spatial and (b) frequency domains.

sampling $f(x, y)$ by means of the two-dimensional grid defined by $r_{p q}(x, y)$ [22]. Hence, if the rate and direction of variation of the original signal $f(x, y)$ are similar to those of $r_{p q}(x, y)$, the magnitude of $T_{p q}$ will be larger.

Each Tchebichef kernel $r_{p q}(x, y)$ defines a sampling grid for the computation of $T_{p q}$. However, for a given order $s$, the grids derived from the corresponding Tchebichef kernels are closely related. These kernels sample the image according to the same pattern of positive and negative pulses. Nevertheless, the orientation of this pattern varies from one kernel to another. Hence, $s$-order Tchebichef kernels $r_{p q}(x, y)$ represent rotated versions of the same sampling grid, capturing the same pattern of variation in the image at different orientations.

To illustrate this, Fig. $\underline{2}$ depicts three different Tchebichef kernels of order $s=4: r_{0,4}(x, y), r_{1,3}(x, y)$, and $r_{2,2}(x, y)$. For each of them, the first picture corresponds to the kernel amplitude. In the second one, local maxima (white) and 

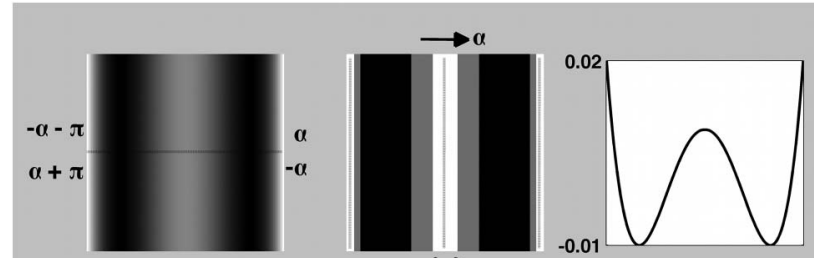

(a)
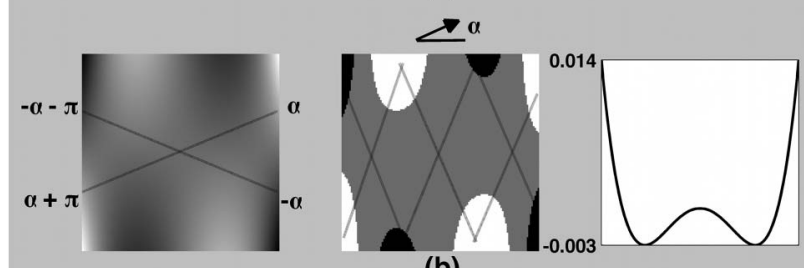

(b)
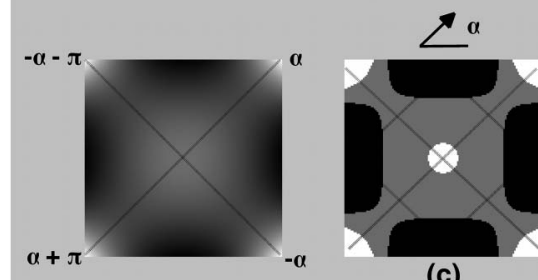

(c)

Fig. 2. Spatial analysis of Tchebichef kernels of order $s=4$ (a) $r_{0,4}(x, y)$, (b) $r_{1,3}(x, y)$, and (c) $r_{2,2}(x, y)$.

minima (black) of the kernel are highlighted in order to identify the orientation $(\alpha)$ of the sampling grid. Finally, the third image depicts the profile of the kernel along the direction indicated by $\alpha$. For this analysis, the orientation of $r_{0,4}(x, y)$ will be taken as reference ( $\alpha=0 \mathrm{rad}$ ). The orientation of a kernel (grid) is given by the arrangement of positive and negative pulses, so that $\alpha$ is normal to the segment connecting consecutive pulses of the same sign. Thus, the kernel $r_{0,4}(x, y)$ detects the varying pattern given by its profile at $\alpha=0 \mathrm{rad}$. As can be observed, the kernel $r_{1,3}(x, y)$ implements a rotated version $(\alpha>0)$ of the sampling grid defined by $r_{0,4}(x, y)$. As a result, the kernel $r_{1,3}(x, y)$ seeks to identify the same pattern of variation as $r_{0,4}(x, y)$ at a distinct orientation. This behavior is reflected by the profile of $r_{1,3}(x, y)$ along $\alpha$, which varies as that of $r_{0,4}(x, y)$. According to the alignment of positive and negative pulses in the $r_{1,3}(x, y)$ polynomial, its orientation $(\alpha)$ is between 0 and $\pi / 4 \mathrm{rad}$. Furthermore, it is worth noting that the directions represented by $\alpha+\pi,-\alpha$, and $-(\alpha+\pi)$ are also explored. The same phenomenon can be observed for the kernel $r_{2,2}(x, y)$, which implements the target pattern of variation at the direction given by $\alpha=\pi / 4 \mathrm{rad}$. Additionally, similar conclusions are extracted from the analysis of the other two kernels of order $s=4$ not included in the figure: $r_{3,1}(x, y)$, for which $\alpha$ is between $\pi / 4$ and $\pi / 2 \mathrm{rad}$, and $r_{4,0}(x, y)$, which corresponds to $\alpha=\pi / 2 \mathrm{rad}$.

It must be appreciated that, for $s>N-1$, the patterns implemented by the corresponding kernel functions are searched in other directions than $k \pi / 2 \mathrm{rad}$, where $k$ is any integer. The reason is that these angles determine the shortest image sections and, thus, the varying patterns associated with these kernels cannot occur along them (i.e., there are no sufficient image samples along these directions to capture the patterns). Therefore, the search is performed at other directions for which the length of the image section is large enough. For instance, for the maximum order $s=2 N-2$, the corresponding varying pattern can only be found along the longest section of the image, which corresponds to an orientation of $\alpha=\pi / 4 \mathrm{rad}$.

From this analysis, the magnitude of $T_{p q}$ evaluates the occurrence of a determined spatially varying pattern, which is specified by the order $s=p+q$. The direction at which this pattern is observed depends on $p$ and $q$. If $p<q$, directions in the range $(-\pi / 4, \pi / 4) \cup(3 \pi / 4,5 \pi / 4)$ rad are explored. For $p>q$, these are in the limits given by $(\pi / 4,3 \pi / 4) \cup(5 \pi / 4,7 \pi / 4) \mathrm{rad}$. Finally, in the special case $p=q$, the kernel is oriented at $(2 k-1) \pi / 4 \mathrm{rad}$, where $k$ is any integer. Hence, the feature $M(s)$ quantifies the occurrence of the pattern given by $s$ in the underlying image. The oscillating profile of the patterns implemented by Tchebichef kernels makes $M(s)$ a useful measure to characterize texture.

\section{Spectral Analysis of Tchebichef Moments}

Spectral properties of Tchebichef kernels also provide useful information for the interpretation of the feature $M(s)$. For this purpose, the starting point is considering each kernel $r_{p q}(x, y)$ as a filter. Hence, the signal $g_{p q}(x, y)$ results from filtering the original image $f(x, y)$ using $r_{p q}(x, y)$ :

$$
\begin{aligned}
g_{p q}(x, y) & =f(x, y) * r_{p q}(x, y) \\
& =\sum_{i=0}^{N-1} \sum_{j=0}^{N-1} f(i, j) r_{p q}(x-i, y-j) .
\end{aligned}
$$

According to symmetric properties of discrete Tchebichef polynomials described in [21] , the kernel $r_{p q}(x, y)$ will be even symmetric if its order $s=p+q$ is even while it will be odd symmetric when the order is odd. Therefore, $g_{p q}(x, y)$ can be expressed in terms of the correlation between $f(x, y)$ and $r_{p q}(x, y)$ :

$$
\begin{aligned}
g_{p q}(x, y) & =(-1)^{p+q} f(x, y) \circ r_{p q}(x, y) \\
& =(-1)^{p+q} \sum_{i=0}^{N-1} \sum_{j=0}^{N-1} f(i, j) r_{p q}(i+x, j+y) .
\end{aligned}
$$

The moment $T_{p q}$ is then formulated as a function of $g_{p q}(x, y)$ evaluated at the origin:

$$
T_{p q}=(-1)^{p+q} g_{p q}(0,0)
$$

Additionally, using the convolution theorem, $g_{p q}(x, y)$ can be written in terms of $F(u, v)$ and $R_{p q}(u, v)$, which denotes the discrete Fourier transform (DFT) of $f(x, y)$ and $r_{p q}(x, y)$, respectively. The following result is obtained:

$$
\begin{aligned}
g_{p q}(x, y) & =\mathrm{DFT}^{-1}\left\{G_{p q}(u, v)\right\} \\
& =K \sum_{u=0}^{N-1} \sum_{v=0}^{N-1} F(u, v) R_{p q}(u, v) \exp \left[-j 2 \pi\left(\frac{u x+v y}{N}\right)\right],
\end{aligned}
$$

where $G_{p q}(u, v)$ is the DFT of the signal $g_{p q}(x, y)$ and $K$ represents a constant value. By using this expression in the definition of the moment $T_{p q}$ given in Eq. (9), the dependence of $T_{p q}$ on $F(u, v)$ and $R_{p q}(u, v)$ is obtained: 


$$
\begin{aligned}
T_{p q} & =(-1)^{p+q} K \sum_{u=0}^{N-1} \sum_{v=0}^{N-1} G_{p q}(u, v) \\
& =(-1)^{p+q} K \sum_{u=0}^{N-1} \sum_{v=0}^{N-1} F(u, v) R_{p q}(u, v) .
\end{aligned}
$$

Functions $F(u, v)$ and $R_{p q}(u, v)$ can be separated into magnitude and phase components to appreciate their effect on $T_{p q}$. Hence, the moment can be equivalently expressed as

$$
T_{p q}=(-1)^{p+q} K \sum_{u=0}^{N-1} \sum_{v=0}^{N-1}\left|G_{p q}(u, v)\right| \cos \left[\phi_{G_{p q}}(u, v)\right],
$$

where $\quad\left|G_{p q}(u, v)\right|=|F(u, v)|\left|R_{p q}(u, v)\right| \quad$ and $\quad \phi_{G_{p q}}(u, v)=$ $\phi_{F}(u, v)+\phi_{R_{p q}}(u, v)$. Imaginary parts are canceled due to symmetric properties of $\left|G_{p q}(u, v)\right|$ and $\phi_{G_{p q}}(u, v)$. From Eq. (12), the magnitude of $T_{p q}$ is influenced by two conditions. Initially, a larger magnitude is expected if the energy of $f(x, y)$ is concentrated in similar frequency components to $r_{p q}(x, y)$. In addition, increased magnitude will be obtained if $\phi_{G_{p q}}(u, v)$ is either $0, \phi_{F}(u, v)=-\phi_{R_{p q}}(u, v)$, or $\pi, \phi_{F}(u, v)=\pi-\phi_{R_{p q}}(u, v)$, for each point $(u, v)$ in the frequency plane. If the sign of $\cos \left[\phi_{G_{p q}}(u, v)\right]$ recurrently changes, the terms of the summation tend to cancel each other, resulting in a lower magnitude of $T_{p q}$. Thus, its value is expected to be larger if $\cos \left[\phi_{G_{p q}}(u, v)\right]$ tends to preserve its signs, namely $\phi_{G_{p q}}(u, v)$ is either between $(-\pi / 2, \pi / 2)$ or between $(\pi / 2,3 \pi / 2) \mathrm{rad}$.

As expressed in Eq. (12), the spectrum of $r_{p q}(x, y)$ determines those frequency components of $f(x, y)$ that influence the value of $T_{p q}$. Figure 1 reflects that kernels with the same order are concentrated in similar frequencies occurring at different orientations. This result is consistent with the conclusions drawn from spatial analysis, which revealed that, for a given order, all the kernels are characterized by the same varying pattern along distinct directions. The value of $M(s)$ is then computed from a subset of the frequency components of $f(x, y)$, which is defined by the set of kernels $r_{p q}(x, y)$ such that $s=p+q$. Therefore, grouping $s$-order Tchebichef kernels results in a specific frequency band for the computation of $M(s)$. Figure 3 depicts this band (black color identifies components with a higher magnitude of the spectrum) for $s=0, N / 2, N-1,3 N / 2$, and $2 N-2(N=128)$. As can be observed, the combination of kernels with the same order has the effect of a bandpass filter. The filter moves from the origin $(s=0)$ toward the maximum frequency $(s=2 N-2)$ as $s$ is increased, showing the relationship between moment order and frequency. The whole range of values for $s$ defines a filter bank that entirely covers the frequency plane. Thus, the feature $M(s)$ quantifies the response of the original image to each of the bandpass filters in this bank.

The shape of the resulting bandpass filters indicates that the frequency components of $s$-order kernels are not exactly equal (a ring centered around the origin would reflect the same frequency content for any orientation). For example, the frequencies associated with kernels oriented at $\alpha=0$ or $\alpha=\pi / 2$ rad are higher than those corresponding to kernels oriented at any other $\alpha$ between these two values. This result is in agreement with the observation of the kernels in the spatial domain. For $\alpha=k \pi / 2$, where $k$ is any integer and the order is assumed to be $s \leq N-1$, a specific pattern of variation occurs along the shortest spatial lengths in the

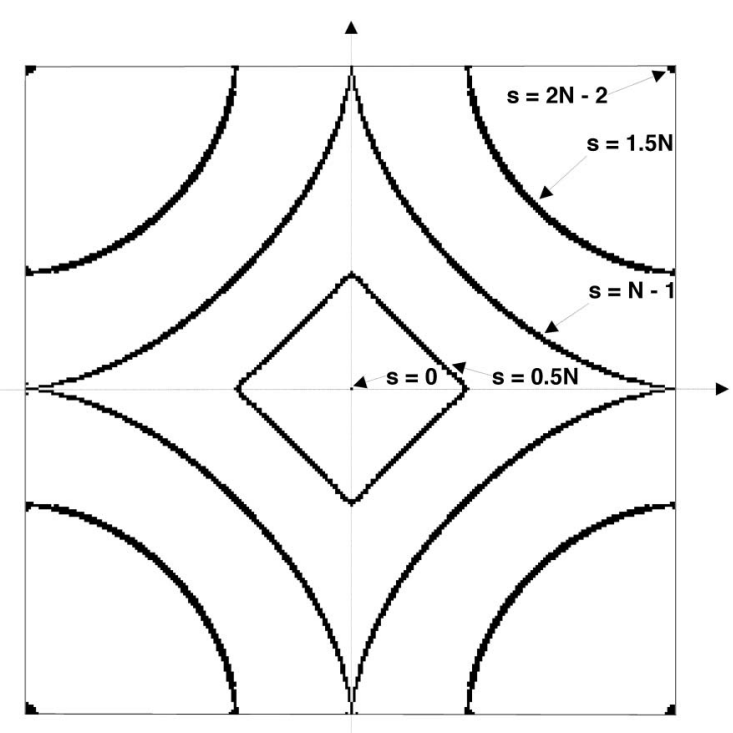

Fig. 3. Bandpass filters resulting from the combination of $s$-order Tchebichef kernels (highlighted bands correspond to frequency components with higher energy content).

image. However, for other orientations, the same pattern is found along longer image sections, resulting in lower rates of variation. Specifically, if $\alpha=k \pi / 4$, the target pattern is found along the longest possible image section. As the same pattern occurs along a different spatial length, distinct frequencies are associated with each kernel of order $s$, leading to the observed bandpass filters.

\section{Application to Texture Characterization}

The analysis of Tchebichef polynomials reveals that the value of $M(s)$ assesses the occurrence of patterns similar to those associated with kernels of order $s$. The profile of these kernels, characterized by an oscillating behavior, suitably adapts to texture analysis. Texture images are composed of characteristic elements that are repeated in the space [1] ]. Thus, the texture surface is expected to show recurrent patterns of variation along space coordinates. Due to this repetitive behavior, these patterns can be captured through the correlation of the image with the set of Tchebichef kernels. The feature $M(s)$ measures this correlation taking into account different directions in the image. In other words, $M(s)$ encodes those patterns characterizing the texture and, thus, different $M(s)$ signatures are expected for distinct texture types.

It is worth noting that the number of kernels that contribute to $M(s)$ depends on $s$. This number is given by $s+1$, if $0 \leq s \leq N-1$, or $2 N-1-s$, if $N-1<s \leq 2 N-2$. However, there is no bias in the computation of the feature $M(s)$. The number of $s$-order kernels is such that it is sufficient to capture the fluctuating pattern specified by $s$ at any orientation. This is reflected by the interpretation of $M(s)$ in the frequency domain. As $s$ increases from 0 to $N-1$, a higher number of kernels is required to cover the entire range of orientations at which the target pattern may occur, i.e., additional filters (kernels) are needed to cover the region of the frequency plane that corresponds to $s$. For $s>N-1$, the target pattern cannot appear at all the possible orientations in the image. Therefore, a smaller number of kernels is required to capture its occurrence (see Figs. 1 and 3 ). 
On the other hand, the proposed $M(s)$ feature is not strictly invariant to rotation. This result is drawn from the bandpass filters defined by the set of $s$-order kernels. In order to be insensitive to rotation, ring-shaped filters centered on the origin should be obtained. This is not the case for the set of bandpass filters from which $M(s)$ is computed. As a result, a different $M(s)$ will be expected for rotated versions of the same image. For example, a fluctuating pattern oriented at $0 \mathrm{rad}$ and exhibiting a profile similar to that of the $a$-order kernel $r_{0 a}(x, y)$ will be reflected by a higher value of $M(s)$ at $s=a$. If the original image is rotated $\alpha \mathrm{rad}$, with $0<\alpha<\pi / 2 \mathrm{rad}$, the pattern will be aligned along the orientation given by $\alpha$. From our previous analysis, this pattern will be captured by kernels with order $s=a^{\prime}\left(a^{\prime}>a\right)$. The reason is that the pattern will preserve its frequency content, that is, its rate of variation, while the $a$-order kernel $r_{p q}(x, y), a=p+q$, oriented at $\alpha$, is characterized by smaller frequencies than the $a$-order kernel $r_{0 a}(x, y)$ at $0 \mathrm{rad}$. Therefore, for the rotated image, the influence of this pattern will be reflected by $M^{\prime}(s)$ at $s=a^{\prime}\left(\alpha^{\prime}>a\right)$. After rotation, the pattern will be correlated with the $a^{\prime}$-order kernel $r_{p^{\prime} q^{\prime}}(x, y), a^{\prime}=p^{\prime}+q^{\prime}$, with a rate of variation higher than that of $r_{p q}(x, y)$ along the direction specified by $\alpha$. However, small differences between signatures $M(s)$ and $M^{\prime}(s)$ are expected if the patterns that compose the texture are not aligned at a specific orientation. If there are patterns randomly oriented between 0 and $\pi \mathrm{rad}$, these will tend to be rearranged at closely oriented directions after rotating the image. Therefore, for textures characterized by patterns without a marked orientation, the proposed feature will be less influenced by rotation, achieving robustness to this transformation. As will be shown, our experimental results confirmed this effect.

\section{B. Conventional Methods for Texture Analysis}

Three conventional methods for texture analysis were considered for comparison purposes with the presented DTM-based procedure. These include Haralick's GLCM, GF, and LBP. These methods have proven to be successful for texture classification and are widely recognized by the scientific community in the field [1,39]. A detailed explanation of the basis for GLCM, GF, and LBP can be found in [36], [37,38], and [39], respectively. Here, we provide a brief description of the texture features derived from each of these methods.

\section{Haralick's GLCM}

The GLCM was computed for a quantization level $L_{g}=8$ [ [5] . Four different matrices were obtained by varying the configuration of the displacement vector, which is specified by the distance $(d)$ and the angle $(\theta)$. The value of $d$ was set to 1 while angles $0,45,90$, and 135 deg were assessed [3]. As suggested in [3] 10 statistical features were computed from each of the four co-occurrence matrices: energy, contrast, correlation, homogeneity, entropy, autocorrelation, dissimilarity, cluster shade, cluster prominence, and maximum probability. Thus, a total of 40 descriptors (10 statistical features for each of the four orientations) were obtained from the texture.

\section{2. $G F$}

The results reported by Bianconi and Fernández [40] were considered for the design of the GF bank. Hence, a total of 24 filters, four different central frequencies and six orientations, were used. The maximum value for the central frequency of a filter was set to $0.327 \mathrm{~Hz}$ and a ratio between consecutive frequencies of half-octave was applied. The standard deviation of the Gaussian filter along both radial and angular directions was set to 0.5. As suggested in [40], mean and standard deviation from the magnitude of the filtered images were used as texture features. As a result, a feature vector composed of 48 elements (two features per filter and a total of 24 filters) was used to describe the texture.

\section{3. $L B P$}

The rotation-invariant uniform operator $\left(\mathrm{LBP}_{P, R}^{\text {riu2 }}\right)$ was considered to obtain the LBP code of an image. As suggested by Ojala et al. [39], the number of neighbors $(P)$ around a central pixel and the radius of the circumference on which they are distributed were set to 24 and 3 , respectively. Thus, every pixel in the original image was then assigned one of $P+2$ possible values. Each of them represents a different structuring element. A histogram representing the relative frequency of occurrence for each of the $P+2$ structures was used as the texture feature vector. The number of bins, $P+2$, determines the dimension of this vector.

\section{RDA}

A multivariate feature vector $\mathbf{z}=\left(z_{1}, \ldots, z_{l}\right)$ is obtained from the analysis of an image using one of the described methods: DTM, GLCM, GF, and LBP. This vector summarizes the information in the texture according to the properties exhibited by the corresponding method. Multivariate analysis is required to classify it into one of several categories. In this study, supervised classification is proposed using RDA [32].

RDA generalizes the concept of discriminant analysis (DA), including quadratic DA (QDA) and linear DA (LDA) as special cases. It addresses the problem of covariance matrix estimation in high dimensional spaces. Covariance matrix estimates $\left(\Sigma_{k}\right)$ become highly variable when the number of samples in each category is small compared with the dimension of the input feature space. As a result, the output of the DA classifier may be biased by the smallest eigenvalues. Friedman suggested a method to prevent this problem based on regularization techniques [32]. The idea is to improve the estimates of the covariance matrices by reducing the variance associated with the sample-based estimate while increasing the bias. This adjustment is determined by two parameters $\lambda$ and $\mu(0 \leq \lambda, \mu \leq 1)$ as expressed by the following equation:

$$
\sum_{k}(\lambda, \mu)=(1-\mu) \sum_{k}(\lambda)+\frac{\mu}{l} \operatorname{trace}\left[\sum_{k}(\lambda)\right] I,
$$

where $l$ is the dimension of the input feature space, $I$ denotes the identity matrix, and

$$
\sum_{k}(\lambda)=\frac{(1-\lambda) \mathbf{S}_{k}+\lambda \mathbf{S}}{(1-\lambda) N_{k}+\lambda N_{T}} .
$$

The terms $\mathbf{S}$ and $\mathbf{S}_{k}$ are, respectively, given by $\mathbf{S}=\sum_{k=1}^{N_{C}} \mathbf{S}_{k}$ and $\mathbf{S}_{k}=\sum_{j=1}^{N_{k}}\left(\mathbf{z}_{j}-\overline{\mathbf{z}}_{k}\right)\left(\mathbf{z}_{j}-\overline{\mathbf{z}}_{k}\right)^{T}$, where $N_{C}$ is the number of categories, $N_{k}$ is the number of samples in the $k$ th category such that $N_{T}=\sum_{k=1}^{N_{C}} N_{k}$, and $\overline{\mathbf{z}}_{k}$ is the mean vector of the samples in that category.

The parameter $\lambda$ controls the choice between QDA $(\lambda=0)$ and LDA $(\lambda=1)$. On the other hand, $\mu$ controls the effect of decreasing larger eigenvalues while increasing smaller ones. 
As a result, the bias derived from the sample-based estimation of the covariance matrix (represented by $\mathbf{S}_{k}$ ) is removed [32]. There is no previous knowledge about the most appropriate value for these regularization parameters. Thus, a wide set of combinations of $\lambda$ and $\mu$ were evaluated by varying them from 0 to 1 with steps of 0.1 . The configuration with the highest performance was retained as the optimum.

\section{DATA AND EXPERIMENTAL RESULTS}

Texture images from Brodatz [33], Outex [34], and VisTex [35] databases were employed for a comparative analysis between the proposed DTM-based method and the three conventional techniques used as reference: GLCM, GF, and LBP. Different experiments were conducted from the selected texture images. A description of the proposed experiments and the achieved results is provided in this section.

\section{A. Public Databases}

\section{Brodatz Database}

Thirteen textures from the Brodatz album included in the image database of the Signal and Image Processing Institute at the University of Southern California [33] were processed in our experiments: bark (D12), brick (D94), bubbles (D112), grass (D9), leather (D24), pigskin (D92), raffia (D84), sand (D29), straw (D15), water (D38), weave (D16), wood (D68), and wool (D19). The number in parenthesis indicates the page number of the Brodatz texture book where the original image can be found. A set of seven grayscale images are available for each of these textures depending on their orientation: 0,30 , $60,90,120,150$, and $200 \mathrm{deg}$. Each of them has a size of $512 \times 512$ pixels $(8$ bits per pixel).

\section{Outex Database}

The Outex database [34] contains 320 color texture images. Each of them was captured using six spatial resolutions $(100,120,300,360,500$, and $600 \mathrm{dpi})$ and nine rotation angles $(0,5,10,15,30,45,60,75$, and $90 \mathrm{deg})$. Images are stored in RGB format with a resolution of 24 bits and a size of $538 \times 746$ pixels. In our study, 29 textures (the first one in each category) were selected from the Outex database: barleyrice001, canvas001, cardboard001, carpet001, chips001, crushedstone001, flakes001, four001, foam001, fur001, granite001, granular001, gravel001, groats001, leather001, mineral001, paper001, pasta001, pellet001, plastic001, quartz001, rubber001, sand001, sandpaper001, seeds001, tile001, wallpaper001, wood001, and wool001. The luminance channel was extracted to obtain gray-scale images with a resolution of 8 bits per pixel. Images with a spatial resolution of $100 \mathrm{dpi}$ and horizon sunlight illumination were selected from the database for our experiments. Nine different images were extracted for each texture by including all the available orientations.

\section{VisTex Database}

The VisTex database [35] provides a set of texture images that are representative of real-world scenarios. In our study, images labeled as "reference textures" in the VisTex database were considered. These textures are grouped depending on the object represented in the image: bark, grass, water, brick, clouds, etc. They are stored in RGB format with a resolution of 24 bits. In our study, color information was discarded, converting them to gray-scale texture images. A subset of
20 images of size $512 \times 512$ from this database was evaluated in our experiments: bark0006, brick0000, brick0004, clouds0001, fabric0013, fabric0017, flowers0006, food0000, food0001, grass0001, leaves0012, metal0002, metal0004, misc0001, misc0002, sand0000, sand0002, tile0008, water0005, and wood0002. It is worth remarking that the same subset was used by other researchers for the evaluation of other texture analysis techniques [8].

\section{B. Results}

Several experiments were conducted using texture images from Brodatz, Outex, and VisTex datasets. A common methodology was adopted for a fair comparison between DTM, GLCM, $\mathrm{GF}$, and LBP. It consists in feature extraction using one of these methods and classification of the resulting feature vector by means of RDA. In our experiments, the influence of the evaluated dataset, the image size, and the orientation of the texture on classification performance was assessed.

\section{Classification of Nonrotated Textures}

The first set of experiments involves textures with a fixed orientation. Brodatz and Outex datasets contain texture samples captured at different angles. Thus, those textures with a rotation of 0 deg were selected. In the case of the VisTex dataset, all the available textures were included in this set of experiments as they each have a unique orientation. As a result, three different datasets were used in the first phase of the experiments: 13 Brodatz textures $(512 \times 512), 29$ Outex textures $(746 \times 538)$, and 20 VisText textures $(512 \times 512)$. For the three datasets, one texture image was available per each category. The utility of the proposed feature extraction methods was separately evaluated on these datasets. For each of them, four different classification tasks were defined depending on the size of the patch to be processed: $128 \times 128,64 \times 64$, $32 \times 32$, and $16 \times 16$ pixels.

The holdout approach was used to estimate the performance of DTM, GLCM, GF, and LBP [24]. Independent training and test sets were generated by dividing the original texture image in each category into two nonoverlapping subimages of size $256 \times 512,373 \times 538$, and $256 \times 512$ pixels for textures from Brodatz, Outex, and VisTex datasets, respectively. One of these subimages was used to extract training samples while the other was allocated for testing. Overlapped texture regions were extracted from each of them to obtain sufficiently large training and test sets. For all the image sizes considered in the experiments, the training set was composed of texture patches defined by a sliding window with an overlapping of $75 \%$ on the training subimage. Overlapping was minimized to obtain patches in the test set in order to prevent bias in classification results. Thus, $50 \%$ overlapping was used to generate test patches of size $128 \times 128$ and $64 \times 64$ pixels while $25 \%$ and $0 \%$ overlapping was used for extracting texture patches of size $32 \times 32$ and $16 \times 16$ pixels, respectively.

For each classification task, a RDA classifier was implemented from data in the training set. Subsequently, the accuracy achieved on the test set was computed. It was estimated as the ratio between the number of texture patches correctly classified and the total number of samples. The highest classification accuracy from all the evaluated pairs of parameters $\lambda$ and $\mu$ was retained. Table 1 summarizes the experimental setup and the classification accuracy achieved 
by the evaluated methods. As can be observed, the most effective method depends on the dataset and the patch size. GLCM and GF achieved the highest performance on Brodatz textures while the proposed DTM-based method outperformed the others when images from the Outex dataset were processed. On the other hand, smaller differences are appreciated on VisTex textures. This dataset showed to be the least difficult as DTM, GLCM, and GF provided high accuracy even for reduced texture size.

For all the methods assessed in the study, accuracy decreased as the size of the texture patch was reduced. This was an expected behavior as textures are characterized by the repetition of patterns in the spatial domain [1]. Thus, small patches may not capture a sufficient number of these patterns, i.e., low frequency resolution is obtained. The performance decrease due to this effect was specially marked for LBP. This is motivated by the estimation of the histogram representing the relative frequency for each structure identified by the uniform LBP patterns. The number of LBP pattern samples is limited by the dimension $N \times N$ of the texture under analysis. As this dimension is reduced, a smaller number of samples is available to compute the histogram, resulting in a poor estimation of the statistical behavior of these patterns and, thus, of the texture.

The method based on DTM has been demonstrated to provide useful information about texture since high classification accuracy was achieved on the proposed problems. An index of separability (SI) was computed for each element of $M(s)$ to determine the range of $s$ at which textures show higher differences. This index was obtained as the ratio between interclass $\left(\sigma_{B}^{2}\right)$ and intraclass variabilities $\left(\sigma_{W}^{2}\right)$ [41]. For a one-dimensional variable $z$, the SI is computed as follows:

$$
S I=\frac{\sigma_{B}^{2}}{\sigma_{W}^{2}}=\frac{\sum_{k=1}^{N_{C}} N_{k}\left(\bar{z}_{k}-\bar{z}\right)^{2}}{\sum_{k=1}^{N_{C}} \sum_{i \in C_{k}}\left(z_{i}-\bar{z}_{k}\right)^{2}},
$$

where $C_{k}\left(k=1, \ldots, N_{C}\right)$ denotes the $k$ th category, $N_{k}$ is the number of samples in $C_{k}, \bar{z}_{k}$ is the mean value of $z$ in $C_{k}$, and $\bar{z}$ is the global mean value of $z$ in the available sample set. Thus, higher SI indicates that classes can be more easily separated using linear boundaries.

Table 1. Classification of Nonrotated Textures ${ }^{a}$

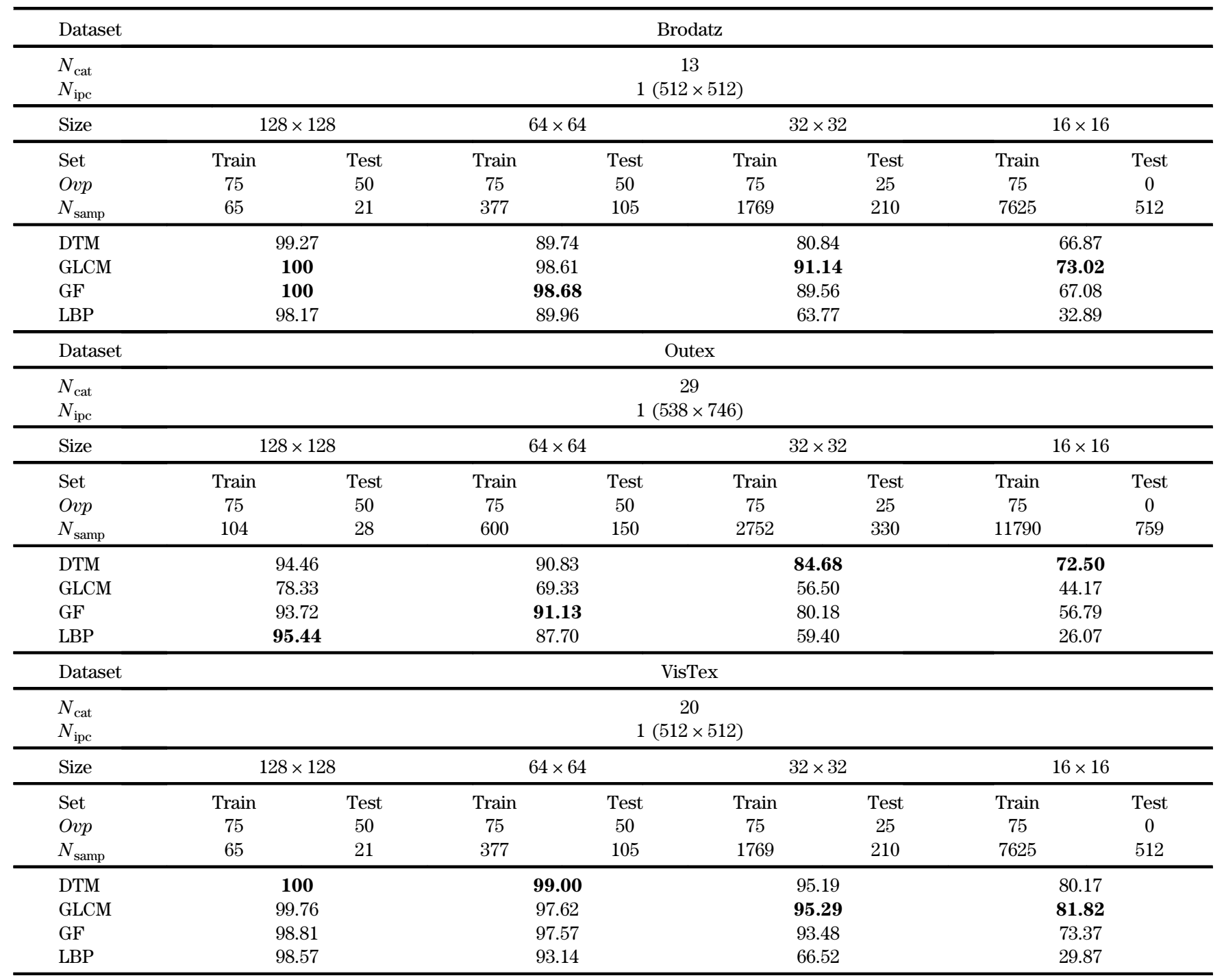

${ }^{a} N_{\text {cat }}$ : number of distinct categories in the classification problem; $N_{\text {ipc }}$ : number of available images per category; Ovp: overlapping percentage between adjacent samples; $N_{\text {samp }}$ : number of samples. 

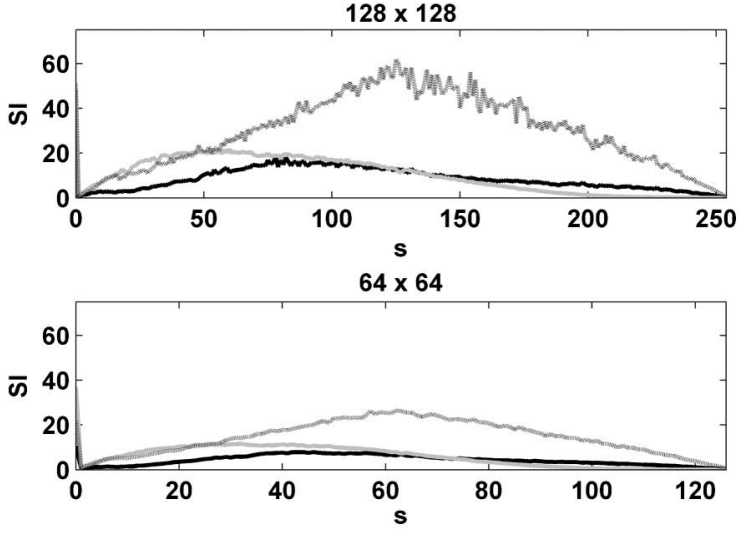

$32 \times 32$

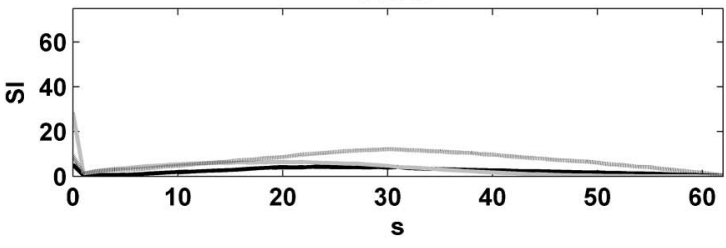

$16 \times 16$

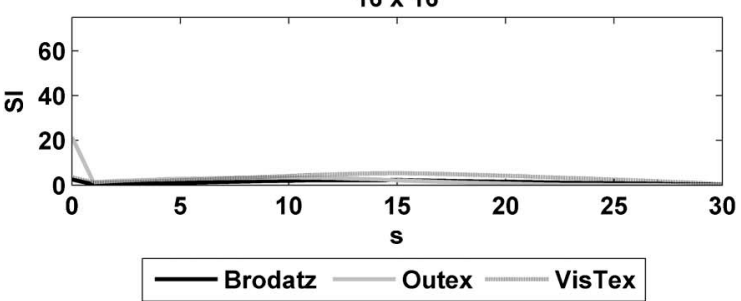

Fig. 4. Evolution of the SI of coefficients $M(s)$ as a function of the moment order for Brodatz, Outex, and VisTex datasets.
Figure $\underline{4}$ depicts the dependence of SI on the moment order $s$ for Brodatz, Outex, and VisTex textures. As shown by classification accuracy, the discriminant power decreases as the texture is smaller. In addition, the plot reflects that higher separability between categories relies on the first half of $s$ values in the case of Outex textures while the curve is approximately symmetric for Brodatz and VisTex images. Thus, most of the information representing distinctive texture attributes is contained at intermediate order values.

\section{Classification of Rotated Textures}

The robustness of the four feature extraction methods to texture rotation was evaluated in the second set of experiments. These were carried out only from images in Brodatz and Outex datasets since they provide originally rotated textures. The former includes 13 different texture categories. Seven original images are available per category, each of them corresponding to a different orientation. The latter is composed of 29 texture categories and nine different orientations per category. As in the previous experiments, textures from both datasets were processed separately.

The original textures were divided into two nonoverlapping subimages, which were respectively used to generate training and test patches. As in the previous set of experiments, subimages from the Brodatz dataset have a size of $256 \times 512$ pixels while those from the Outex dataset are $373 \times 538$ pixels. For the four patch sizes assessed in the experiments (i.e., $128 \times 128,64 \times 64,32 \times 32$, and $16 \times 16$ pixels), training samples were generated using a sliding window with $50 \%$ overlapping between adjacent regions. In these experiments, no overlapping was used to extract texture patches in the test set.

Table 2 shows the details of the experiments conducted on both Brodatz and Outex rotated textures. As in the previous

Table 2. Classification of Rotated Textures ${ }^{a}$

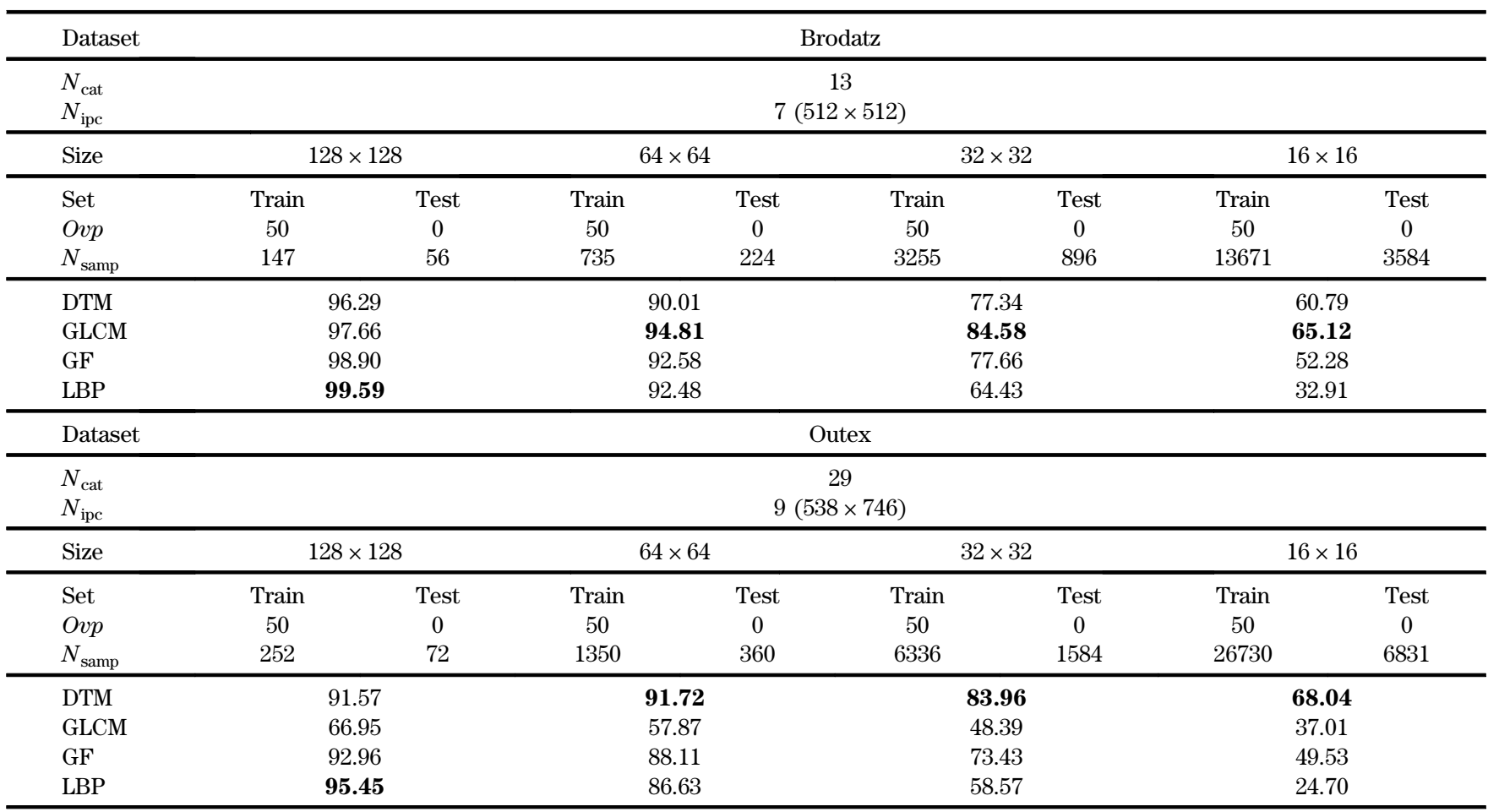

${ }^{a} N_{\text {cat }}:$ number of distinct categories in the classification problem; $N_{\text {ipc }}$ : number of available images per category; Ovp: overlapping percentage between adjacent samples; $N_{\text {samp }}$ : number of samples. 
test, the results show a strong dependence of GLCM on the dataset. Additionally, this second set of experiments reflects that LBP provided the highest classification accuracy when sufficiently large textures $(128 \times 128$ pixels $)$ were considered. However, its performance notably decreased as the patch size was reduced.

The comparison between the results on nonrotated and rotated textures reveals that only LBP achieved similar performance in both cases. In contrast, for the other methods, classification accuracy decreased when rotated textures are processed. This result reflects the rotation invariant property of the evaluated LBP operator [39]. However, it is worth noting that, even though this property is not shared by the other methods, they are also capable of providing reasonable results on rotated textures.

To analyze the robustness of the DTM method against rotation, we evaluated the separability between $M(s)$ vectors from the same texture at different rotation angles. Each texture of the Brodatz dataset has been assessed, taking into account the dependence on the patch size. Thus, a function $\mathrm{SI}(s)$ was obtained for each texture type and for a given size. A small $\mathrm{SI}(s)$ indicates reduced differences between rotated versions of the same texture; i.e., $M(s)$ is not influenced by rotation for the underlying texture. The mean value of the $\mathrm{SI}(s)$ curve was computed. Figure 5 provides the obtained results, which lead to the two following observations. First, SI decreased with the patch size since some information about the texture is lost when a smaller region is observed. Smaller patches tend to be more similar to others derived from the same texture even for different orientations. Second, those textures more influenced by rotation contain structures with a clear orientation. From our results, the highest SI was obtained from samples of the "wood" texture. As can be observed, it is characterized by the repetition of elements with a specific alignment. In contrast, "sand," which contains patterns occurring without a marked orientation, is the texture that showed the highest robustness to rotation (i.e., the smallest SI).

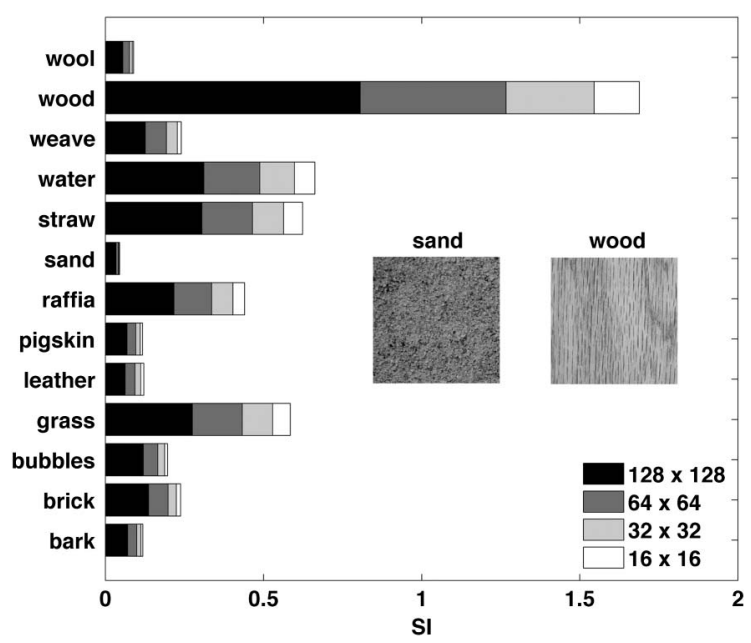

Fig. 5. Analysis of the SI between $M(s)$ curves from the same texture captured at different orientation angles. Results computed from textures in the Brodatz dataset.

\section{DISCUSSION AND CONCLUSIONS}

A novel method for texture characterization based on DTM was presented. A feature vector $M(s)$ is derived from the moment matrix. It represents the response of the texture image to the set of filters implemented by the Tchebichef polynomials of order $s$. To assess the utility of our method, several texture classification problems have been proposed using images from Brodatz, Outex, and VisTex datasets. A comparative analysis was performed by evaluating other conventional approaches for texture analysis such as GLCM, GF, and LBP on the same experiments. The results indicate that the proposed $M(s)$ feature captures essential information about texture, showing its potential for effective texture identification.

Three main conclusions can be drawn from our experiments. First, the method based on DTM represents a valid approach for texture characterization. The performance achieved by our method was comparable to that of GLCM, $\mathrm{GF}$, and LBP, which are recognized by the scientific community in the field as effective approaches to texture analysis $[\underline{1}, \underline{5}, \underline{39}]$. Indeed, the proposed DTM-based method achieved the highest average classification accuracy over all the experiments with a value of $85.66 \%$ whereas it was $76.90 \%, 83.29 \%$, and $69.81 \%$ for GLCM, GF, and LBP, respectively. Second, the DTM-based method has shown to be robust since it yielded reasonable results regardless of the dataset or the texture patch size. It was observed that the performance of other methods highly depended on the image dataset under analysis. Specifically, in the case of GLCM, it provided an average accuracy of $88.12 \%, 57.32 \%$, and $93.62 \%$ for experiments involving Brodatz, Outex, and VisTex images, respectively. These three average values were $82.64 \%, 84.72 \%$, and $93.59 \%$ for the method based on DTM; $84.59 \%, 78.23 \%$, and $90.81 \%$ for GF; and $71.78 \%, 66.75 \%$, and $72.03 \%$ for LBP. These methods were less influenced by the dataset than GLCM, which markedly failed on Outex textures. This result is motivated by the influence of the dynamic range of the texture on the GLCM method, which is based on the discretization of the original image. If a reduced number of quantization levels $\left(L_{g}\right)$ is used, different textures with a small dynamic range and a similar distribution of their gray-level values will lead to identical GLCM matrices; i.e., every pixel will be assigned the same quantization level. This behavior was observed in some textures from the Outex dataset (fur001, leather001, and rubber001) characterized by small fluctuations of the gray level in a similar interval. On the other hand, for all the evaluated methods, decreasing the size of the texture patch resulted in reduced performance. This is a coherent result since lower frequency resolution is obtained by reducing the image size. However, the size had a less remarkable effect on the DTM-based method. For the five classification problems analyzed in this study, the average reduction in the classification accuracy when considering a size $N=16$ with respect to $N=128$ was of $27.66 \%, 33.32 \%, 38.39 \%$, and 69.99\% for DTM, GLCM, GF, and LBP, respectively. As can be observed, the dependence on the texture patch size is more pronounced in GLCM and GF than in our DTM-based method while it is dramatic in the case of LBP. Third, satisfactory classification accuracy was achieved by means of DTM, GLCM, and GF on rotated textures even though they are not rotation invariant. For a given patch size, the average difference between the accuracy achieved on nonrotated and rotated 
textures was $2.72,7.34$, and 6.46 percentage points for DTM, GLCM, and GF, respectively. These data were computed by comparing the results achieved on nonrotated and rotated textures from Brodatz and Outex datasets. DTM, GLCM, and GF reflected a higher dependence on this effect than $\mathrm{LBP}$. The latter showed similar performance on both nonrotated and rotated textures (the average difference was 0.99 percentage points) since it is the only method that is invariant to rotation. However, for most of the textures, there is not a clear orientation of the structures characterizing them. Therefore, rotation does not have a marked effect on their attributes. This suggests that the feature vectors extracted from rotated versions of the same image using DTM, GLCM, and GF tend to be similar. In the case of DTM, this was observed by analyzing the influence of rotation on the $M(s)$ vector. It was concluded that higher variability between vectors from the same texture at different rotation angles is found when the texture is characterized by structures with a dominant orientation.

Previous techniques for texture characterization based on DTM, or on the closely related DCT, substantially differ from the methodology described in the present study. Indeed, only low-order moments were considered as texture features [28,29]. Nevertheless, according to our results, a more accurate description of texture properties can be obtained by analyzing the whole range of moment orders. The full set of DTM is required for exact image reconstruction, and thus it is worthwhile to consider each moment for the complete description of texture. Moments of increased order capture higher-frequency components characterizing the texture. Therefore, these moments contain valuable information in the case of textures distinguished by rapidly varying patterns. The relevance of the moment order in a given classification task was quantified by the SI, which reflected those components of $M(s)$ with higher discriminant capability. In particular, a higher SI was associated with intermediate values of $s$, proving that moments with a certain order may be essential for texture identification.

On the other hand, the methodology proposed for the computation of the texture signature $M(s)$ could be extended to other bases sharing the properties exhibited by Tchebichef kernels. Therefore, the value of $M(s)$ would be derived from the coefficients of the signal in a different transformed space. The same interpretation of $M(s)$ would be valid provided that the new kernels show similar varying patterns to those of Tchebichef polynomials. The DCT is a special case as its basis functions can be derived from Tchebichef functions [30]. Nonetheless, further analysis is required to determine those kernels that could replace DTM. Additionally, the influence of the chosen kernel on the texture classification performance should be studied.

Several limitations can be found in our methodology. As indicated, the proposed $M(s)$ signature provides a compact global representation of texture properties. Nevertheless, the dimension of the resulting pattern $(2 N-1)$ may be too large for adequate statistical modeling. For high values of $N$, overfitting may arise when using complex classifiers, such as neural networks [24]. The dimension of $M(s)$ is expected to be higher than that of the feature vectors derived from GLCM, $\mathrm{GF}$, and LBP. It is worth noting that the dimension of $M(s)$ is given by the nature of the descriptor; that is, once the image size $(N)$ is fixed, there are no design issues that may influence the dimension of the feature vector, as occurs in the other evaluated methods. Higher performance might have been achieved for GLCM, GF, and LBP by performing an exhaustive search for their optimum design parameters. However, this optimization process is not the purpose of the present research, and common configurations of these methods reported in previous studies were adopted for our experiments $[3,39,40]$. It must be emphasized that several texture databases were evaluated in our study to obtain a global unbiased estimation of the performance of each method. Additionally, RDA was selected as a simple classification approach to prevent overfitting due to the curse of dimensionality [24]. As a result, no design decisions that may bias the conclusions derived from our experiments were taken. On the other hand, our method is not invariant to image transformations. Although it achieved reasonable performance on rotated textures, invariant properties must be addressed in further developments of the method. They are a crucial issue in order to deal with a wider variety of pattern recognition problems. In this context, radial Tchebichef moments were proposed to obtain rotation invariant descriptors from images [42]. Additionally, translation and scale invariants from DTM have been defined [43].

In summary, we propose a new method for texture characterization based on DTM. An exhaustive validation process was carried out by comparing its performance in several texture classification problems with other standard methods for texture analysis. From our experiments, it was demonstrated that the DTM-based method captures essential information about texture, showing comparable or even higher performance than conventional procedures. Additionally, it is worth noting that the proposed $M(s)$ vector can be efficiently obtained through computational strategies developed for DTM [21]. Therefore, we conclude that our method can be considered as an efficient tool to be used in image processing problems involving texture classification.

\section{ACKNOWLEDGMENTS}

J. Víctor Marcos is a Juan de la Cierva research fellow funded by the Spanish Ministry of Economy and Competitiveness.

\section{REFERENCES}

1. M. Tuceryan and A. K. Jain, "Texture analysis," in The Handbook of Pattern Recognition and Computer Vision, C. H. Chen, L. F. Pau, and P. S. P. Wang, eds. (World Scientific, 1993), 235-276.

2. V. S. Bharathi and L. Ganesan, "Orthogonal moments based texture analysis of CT liver images," Pattern Recogn. Lett. 29, 1868-1872 (2008).

3. L. K. Soh and C. Tsatsoulis, "Texture analysis of SAR sea ice imagery using gray level co-occurrence matrices," IEEE Trans. Geosci. Remote Sens. 37, 780-795 (1999).

4. J. Daugman, "How iris recognition works," IEEE Trans. Circuits Syst. Video Technol. 14, 21-30 (2004).

5. T. Randen and J. H. Husoy, "Filtering for texture classification: a comparative study," IEEE Trans. Pattern Anal. Mach. Intell. 21, 291-310 (1999).

6. J. Beck, A. Sutter, and R. Ivry, "Spatial frequency channels and perceptual grouping in texture segregation," Comput. Graph. Image Process. 37, 299-325 (1987).

7. X. Liu and D. Wang, "Texture classification using spectral histograms," IEEE Trans. Image Process. 12, 661-670 (2003).

8. S. Arivazhagan and L. Ganesan, "Texture classification using wavelet transform," Pattern Recogn. Lett. 24, 1513-1521 (2003).

9. A. K. Jain and F. Farrokhnia, "Unsupervised texture segmentation using Gabor filters,” Pattern Recogn. 24, 1167-1186 (1991). 
10. G. M. Haley and B. S. Manjunath, "Rotation-invariant texture classification using a complete space-frequency model," IEEE Trans. Image Process. 8, 255-269 (1999).

11. J. Flusser, T. Suk, and B. Zitová, Moments and Moment Invariants in Pattern Recognition (Wiley, 2009).

12. M. K. Hu, "Visual pattern recognition by moment invariants," IEEE Trans. Inf. Theory 8, 179-187 (1962).

13. J. Bigun and J. M. Hans du Buf, " $N$-folded symmetries by complex moments in Gabor space and their application to unsupervised texture segmentation," IEEE Trans. Pattern Anal. Mach. Intell. 16, 80-87 (1994).

14. M. Wang and A. Knoesen, "Rotation- and scale-invariant texture features based on spectral moment invariants," J. Opt. Soc. Am. A 24, 2550-2557 (2007).

15. R. Mukundan, S. H. Ong, and P. A. Lee, "Image analysis by Tchebichef moments," IEEE Trans. Image Process. 10, 1357-1364 (2001)

16. S. X. Liao and M. Pawlak, "On image analysis by moments," IEEE Trans. Pattern Anal. Mach. Intell. 18, 254-266 (1996).

17. M. R. Teague, "Image analysis via the general theory of moments," J. Opt. Soc. Am. 70, 920-930 (1980).

18. C. H. Teh and R. T. Chin, "On image analysis by the methods of moments," IEEE Trans. Pattern Anal. Mach. Intell. 10, 496-513 (1988).

19. B. Bayraktar, T. Bernas, J. P. Robinson, and B. Rajwa, "A numerical recipe for accurate image reconstruction from discrete orthogonal moments," Pattern Recogn. 40, 659-669 (2007).

20. P. T. Yap, R. Paramesran, and S. H. Ong, "Image analysis by Krawtchouk moments," IEEE Trans. Image Process. 12, 1367-1377 (2003)

21. R. Mukundan, "Some computational aspects of discrete orthonormal moments," IEEE Trans. Image Process. 13, 1055-1059 (2004).

22. C. Y. Wee, R. Paramesran, R. Mukundan, and X. Jiang, "Image quality assessment by discrete orthogonal moments," Pattern Recogn. 43, 4055-4068 (2010).

23. P. T. Yap and P. Raveendran, "Image focus measure based on Chebyshev moments," IEEE Proc. Vis. Image Sig. Proc. 151, 128-136 (2004).

24. C. M. Bishop, Neural Networks for Pattern Recognition (Oxford University, 1995).

25. K. H. Thung, R. Paramesan, and C. L. Lim, "Content-based image quality metric using similarity measure of moment vectors," Pattern Recogn. 45, 2193-2204 (2012).

26. C. Deng, X. Gao, X. Li, and D. Tao, "A local Tchebichef momentsbased robust image watermarking," Signal Process. 89, 1531-1539 (2009).

27. K. Nakagaki and R. Mukundan, "A fast $4 \times 4$ forward discrete Tchebichef transform algorithm," IEEE Signal Process. Lett. 14, 684-687 (2007).
28. B. Li and M. Q. H. Meng, "Computer-aided detection of bleeding regions for capsule endoscopy images," IEEE Trans. Biomed. Eng. 56, 1032-1039 (2009).

29. K. Wu, C. Garnier, J. L. Coatrieux, and H. Shu, "A preliminary study of moment-based texture analysis for medical images," in Proceedings of the 32nd Annual International Conference of the IEEE-EMBS (IEEE, 2010), pp. 5581-5584.

30. K. W. See, K. S. Loke, P. A. Lee, and K. F. Loe, "Image reconstruction using various discrete orthogonal polynomials in comparison with DCT," Appl. Math. Comput. 193, 346-359 (2007).

31. D. G. Sim, H. K. Kim, and R. H. Park, "Fast texture description and retrieval of DCT-based compressed images," Electron. Lett. 37, 18-19 (2001).

32. J. H. Friedman, "Regularized discriminant analysis," J. Am. Stat. Assoc. 84, 165-175 (1989).

33. A. G. Weber, "The ISC-SIPI image database," Tech. Rep. (University of Southern California, 1997).

34. T. Ojala, T. Mäenpää, M. Pietikäinen, J. Viertola, J. Kyllönen, and S. Huovinen, "Outex-new framework for empirical evaluation of texture analysis algorithms," in Proceedings of the 16th International Conference on Pattern Recognition (IEEE, 2002), pp. 701-706.

35. MIT Media Laboratory, "VisTex Vision Texture Database," http:// vismod.media.mit.edu/vismod/imagery/VisionTexture/.

36. R. M. Haralick, K. Shanmugam, and I. Dinstein, "Textural features for image classification," IEEE Trans. Syst. Man Cybern. 3, 610-621 (1973).

37. J. G. Daugman, "Uncertainty relation for resolution in space, spatial frequency, and orientation optimized by two-dimensional visual cortical filters," J. Opt. Soc. Am. A 2, 1160-1169 (1985).

38. J. G. Daugman, "Two-dimensional spectral analysis of cortical receptive fields profile," Vis. Res. 20, 847-856 (1980).

39. T. Ojala, M. Pietikäinen, and T. Mäenpää, "Multiresolution grayscale and rotation invariant texture classification with local binary patterns," IEEE Trans. Pattern Anal. Mach. Intell. 24, 971-987 (2002).

40. F. Bianconi and A. Fernández, "Evaluation of the effects of Gabor filter parameters on texture classification," Pattern Recogn. 40, 3325-3335 (2007).

41. P. Gallinari, S. Thiria, F. Badran, and F. Fogelman-Soulie, "On the relations between discriminant analysis and multilayer perceptrons," Neural Networks 4, 349-360 (1991).

42. R. Mukundan, "A new class of rotational invariants using discrete orthogonal moments," in Proceedings of the 6th IASTED International Conference on Signal and Image Processing (IASTED, 2004), pp. 80-84.

43. H. Zhu, H. Shu, T. Xia, L. Luo, and J. L. Coatrieux, "Translation and scale invariants of Tchebichef moments," Pattern Recogn. 40, 2530-2542 (2007). 